The Astronomical Journal, 120:1192-1197, 2000 September

(C) 2000. The American Astronomical Society. All rights reserved. Printed in U.S.A.

\title{
TWO-COLOR PHOTOMETRY WITH HIGH TEMPORAL RESOLUTION OF THE EXTREMELY VARIABLE BLAZAR PKS 0537-441
}

\author{
Gustavo E. Romero ${ }^{1,2}$ \\ Instituto Argentino de Radioastronomía, C.C. 5, 1894 Villa Elisa, Argentina; romero@irma.iar.unlp.edu.ar \\ Sergio A. Cellone ${ }^{2}$ \\ Facultad de Ciencias Astronómicas y Geofísicas, Universidad Nacional de La Plata, Paseo del Bosque, 1900 La Plata, Argentina; \\ scellone@fcaglp.unlp.edu.ar \\ AND \\ JORGE A. COMBI ${ }^{1,2}$ \\ Instituto Argentino de Radioastronomía, C.C. 5, 1894 Villa Elisa, Argentina; combi@irma.iar.unlp.edu.ar \\ Received 2000 March 31; accepted 2000 May 11
}

\begin{abstract}
We report the results of a microvariability monitoring campaign on the southern blazar PKS 0537-441. The source was observed in the $V$ and $R$ optical bands throughout six consecutive nights. Strong variations at both bands, with amplitudes of $\sim 0.3 \mathrm{mag}$, were observed on timescales of about 2 days. Smaller fluctuations of $\sim 0.05 \mathrm{mag}$ on a scale of a couple of hours are superposed on the larger outburst. The spectral index was also variable during the observing period, with an average value of $\alpha=-1.6$ and changes up to $\sim 27.5 \%$. The spectrum varies in the sense that it becomes harder when the source is brighter, in accordance with predictions of shock-in-jet models. We discuss our results and the possible origin of the variability in this blazar in light of recent higher frequency observations.
\end{abstract}

Key words: BL Lacertae objects: individual (PKS 0537-441) - galaxies: active - galaxies: photometry

\section{INTRODUCTION}

The BL Lacertae object PKS 0537-441 ( $z=0.894$; see Peterson et al. 1976) has shown strong optical variability over timescales of years, displaying erratic fluctuations with amplitudes up to $\Delta m=5 \mathrm{mag}$ (see, e.g., Eggen 1973; Liller 1974; Fan \& Lin 2000). Since the development of CCD photometry, rapid changes in its brightness have been reported by several observers (e.g., Tanzi et al. 1986; Heidt \& Wagner 1996; Romero, Cellone, \& Combi 1999). In particular, Tanzi et al. (1986) observed an increase of $0.4 \mathrm{mag}$ in the $V$ band in just 2 days, whereas Romero et al. (1999) detected changes of $0.2 \mathrm{mag}$ in 24 hours.

At radio frequencies the source is very compact (Tingay et al. 1996) and has shown extreme forms of variability, with fluctuations of $\sim 45 \%$ in its flux density on timescales as short as $\sim 10^{4} \mathrm{~s}$ (Romero, Combi, \& Colomb 1994). Such outbursts, if produced in a synchrotron source, would imply brightness temperatures $T_{b} \sim 10^{21} \mathrm{~K}$, which is 9 orders of magnitude above the inverse Compton limit. The origin of this extreme radio intraday variability has been discussed in terms of interstellar scintillation (Romero et al. 1994), superluminal gravitational microlensing (Romero, Surpi, \& Vucetich 1995; Surpi, Romero, \& Vucetich 1996), and coherent emission processes in the inner jets of the source (Benford \& Lesch 1998). However, it is fair to state that the nature of this kind of variability (observed since then in other objects) is far from clear at present.

At high energies, PKS 0537-441 has also exhibited strong variability (e.g., Tanzi et al. 1986). The source was detected in $\gamma$-rays by the EGRET experiment on board the

\footnotetext{
${ }^{1}$ Member of the Carrera del Investigador Científico, CONICET.

${ }^{2}$ Visiting Astronomer, Complejo Astronómico El Leoncito (CASLEO), operated under agreement between CONICET and the national universities of La Plata, Córdoba, and San Juan, Argentina.
}

Compton Gamma Ray Observatory (Thompson et al. 1993). A flare of a factor $\sim 3$ at $E>100 \mathrm{MeV}$ on a timescale of $\sim 2$ days can be seen in the light curve presented by Hartman (1996).

In this paper, we present the results of an optical campaign on PKS $0537-441$ aimed at determining the characteristics of the variability on very short timescales. We have observed the source with the $2.15 \mathrm{~m}$ CASLEO telescope, obtaining high-quality photometric data in the $V$ and $R$ bands, with a time resolution of $\sim 10$ minutes. In light of our results, we discuss the possible origin of the optical emission in this blazar, and using additional high-energy data, we make some estimates of the mass of the presumed central black hole in the object.

\section{OBSERVATIONS AND DATA ANALYSIS}

The observations were carried out during six consecutive nights in 1998 December with the $2.15 \mathrm{~m}$ CASLEO telescope in San Juan, Argentina. The instrument was equipped with a cryogenically cooled CCD camera with a Tektronix $1024 \times 1024$ chip with a readout noise of $9.6 e^{-}$and a gain of $1.98 e^{-} \mathrm{ADU}^{-1}$. Each field frame contained the blazar and several stars, six of which were used for comparison and control purposes following the procedures described in detail in Romero et al. (1999). We have used the same stars as shown in Figure 1 of that paper.

The microvariability observations were made using Johnson $V$ and Kron-Cousins $R$ filters with integration times of $\sim 100 \mathrm{~s}$. The CCD frames were bias-subtracted and flat-fielded using dome flats with the aim of correcting the pixel-to-pixel variations of the CCD. Standard stars from Landolt's (1992) fields were also observed each night for magnitude calibration.

The data reduction was performed through the IRAF software package running on a Unix workstation. An 8 pixel (6.5) radius aperture was adopted in order to minimize 
the possible contribution of the unresolved host galaxy, in accordance with the recommendations of Cellone, Romero, \& Combi (2000). Differential photometry was then made with the aperture routine APPHOT. As in Romero et al. (1999), comparison and control stars were averaged and the differential light curves calculated as target minus averaged comparison. The advantage of using several averaged stars instead of single ones is discussed in Romero et al (1999). The standard deviation $(\sigma)$ of the comparison minus control averaged stars was adopted as a measurement of the observational errors, as is usual in this kind of study (e.g., Jang \& Miller 1995, 1997). Typical errors are $\sim 0.003 \mathrm{mag}$.

Absolute calibration to the standard system was performed for the BL Lac object and comparison star $C_{1,3}$ (see Fig. 1 in Romero et al. 1999). Since observing conditions during the six nights varied from photometric to partially cloudy, the transformation equations were derived for the best night (December 18) and the rest of the observations were tied to that night by means of differential photometry. A zero-point error of $\sim 0.01 \mathrm{mag}$ is expected for both $V$ and $R$. Standard magnitudes were corrected for Galactic extinction, adopting $E(B-V)=0.038$ (Schlegel, Finkbeiner, \& Davis 1998), and absolute fluxes in the $V$ and $R$ bands were calculated using the calibrations of Bessell (1979).

\section{RESULTS}

\subsection{Light Curves}

The results of the observational campaign are summarized in Table 1, where we list, from left to right, observing bands and periods, errors, the variability timescale of the major outburst [defined as $t_{v} \sim \Delta F /(d F / d t)$ ], the classification of the source as variable or nonvariable according to the $99 \%$ confidence criterion adopted in Romero et al. (1999), the confidence level of the variability, and variability amplitudes, defined as

$$
Y=\frac{100}{\langle D\rangle} \sqrt{\left(D_{\max }-D_{\min }\right)^{2}-2 \sigma^{2}},
$$

where $D_{\max }$ and $D_{\min }$ are the maximum and the minimum in the differential light curve and $\langle D\rangle$ is the averaged value of the light curve over the entire observational campaign.

The light curves at both observing bands are variable at a confidence level of more than $30 \sigma$. The variability amplitudes are $109.2 \%$ and $96.4 \%$ at $V$ and $R$, respectively, showing a fall in brightness of about $0.3 \mathrm{mag}$ in the first two nights, then a comparatively stable period during one night, and a new increase of more than 0.3 mag on the next nights. The differential light curves are shown in Figures 1 and 2.

Intranight results are shown in Table 2, where we list for each night and from left to right the observing band, observational errors, the duration of the observation, variability classification, confidence, and amplitude of the variations. Changes of up to $\sim 33 \%$ occurred within a single night. Figure 3 shows the detailed light curve at both wavelengths
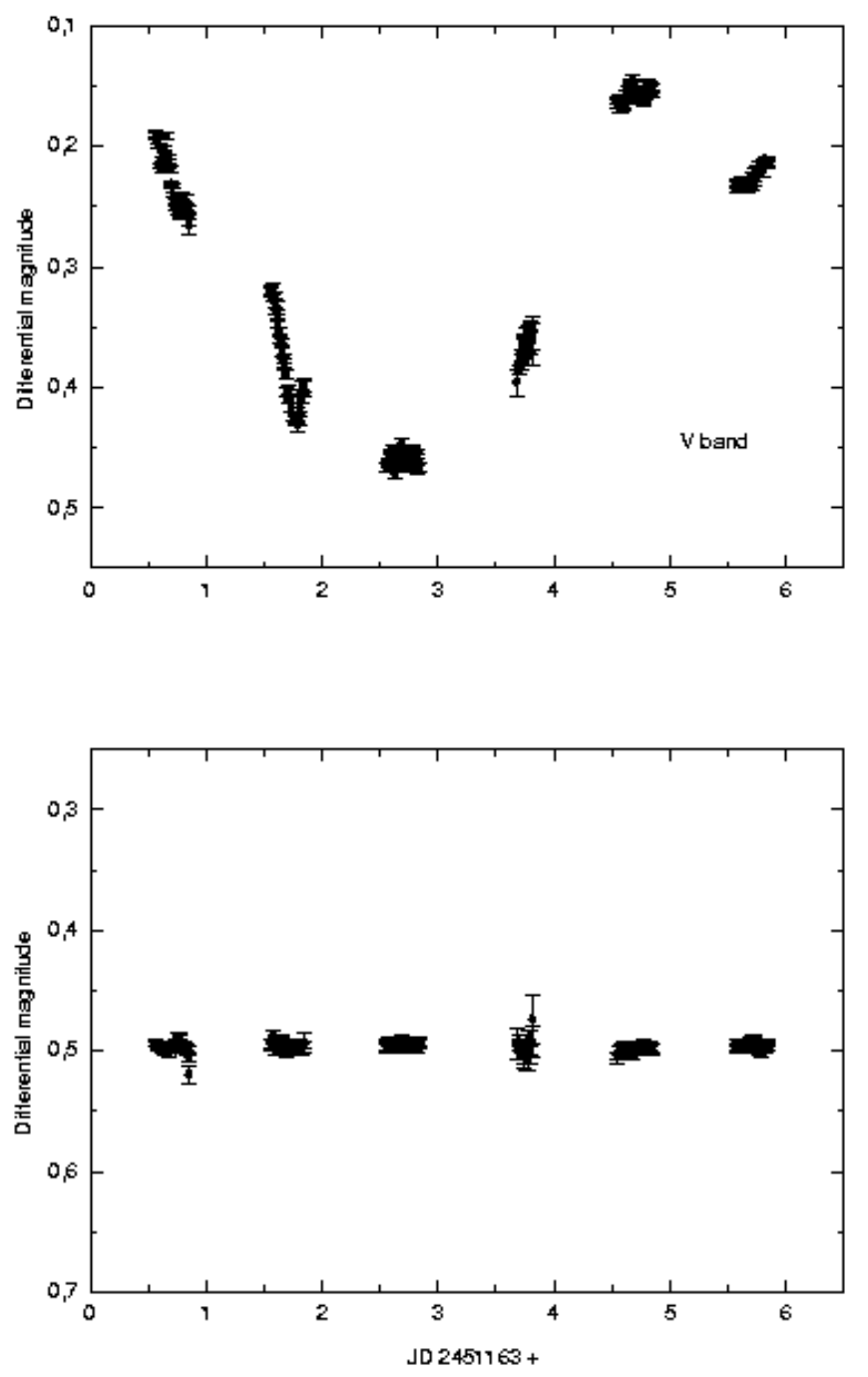

FIG. 1.-Differential light curve for PKS 0537-441 (top) and comparison averaged star (bottom) in the $V$ band for the entire observational campaign.

for the first night of the campaign. Small fluctuations of $\sim 0.05$ mag over timescales $\sim 1 \mathrm{hr}$ are superposed on the general trend in the light curve.

In order to establish whether there is a significant time lag between the light curves at different wavelengths, we have used the interpolated correlation function (ICF) method (Gaskell \& Peterson 1987). The ICF for the entire campaign and for the first night of observation are shown in Figures 4 and 5, respectively. The functions indicate a clear correlation, with a very small time lag between the light curves (the trend is that shorter wavelengths lead the longer ones). Since the peak is rather broad, we have used Gaussian fits to determine the position of the maximum. For the

TABLE 1

Microvariability Optical ObSERvations of PKS 0537-441: ENTIRE CAMPAigN

\begin{tabular}{lcccccc}
\hline \hline Band & UT Date & $\begin{array}{c}\sigma \\
(\mathrm{mag})\end{array}$ & $\begin{array}{c}t_{v} \\
(\mathrm{hr})\end{array}$ & Variable? & $C=\sigma_{s} / \sigma$ & $\begin{array}{c}Y \\
(\%)\end{array}$ \\
\hline$V \ldots \ldots$ & 1998 Dec 16-21 & 0.0034 & 42.4 & Yes & 31.4 & 109.2 \\
$R \ldots \ldots$ & 1998 Dec 16-21 & 0.0034 & 42.4 & Yes & 33.1 & 96.4 \\
\hline
\end{tabular}



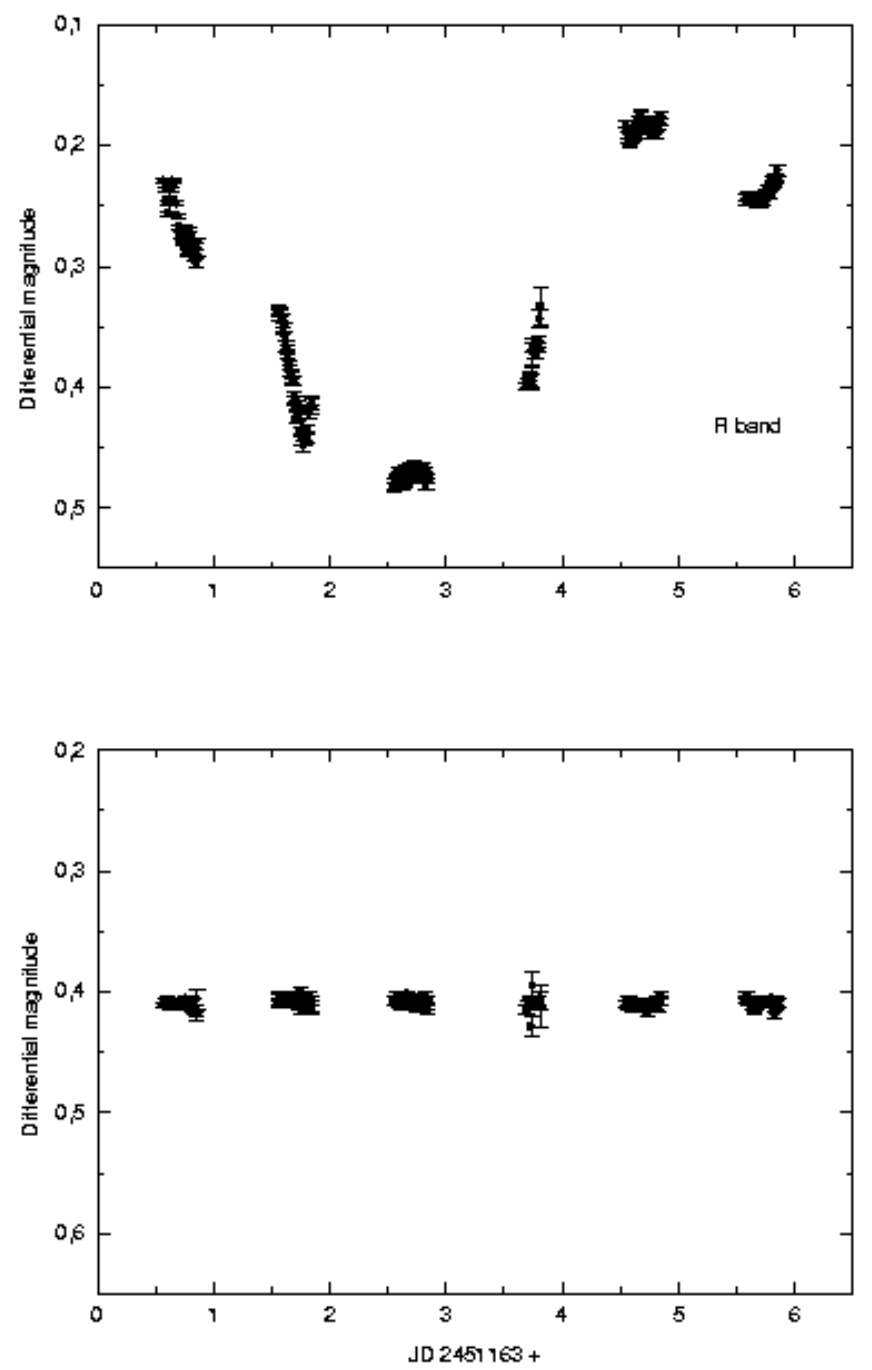

FIG. 2.- Same as Fig. 1, but in the $R$ band

entire campaign, the lag is 17.3 minutes, with an uncertainty of 75.8 minutes. This error is estimated using equation (4) of Gaskell \& Peterson (1987). For the individual nights, the lags are of a few minutes. These very short time lags cannot induce the large variations observed in the spectral index, which occur on much longer timescales (see $\S 3.2$ ).

TABLE 2

INTRANIGHT RESULTS FOR THE OPTICAL OBSERVATIONS OF PKS 0537-441

\begin{tabular}{clccccc}
\hline \hline $\begin{array}{c}\text { UT Date } \\
(1998)\end{array}$ & Band & $\begin{array}{c}\sigma \\
(\mathrm{mag})\end{array}$ & $\begin{array}{c}\Delta t \\
(\mathrm{hr})\end{array}$ & Variable? & $C=\sigma_{\mathrm{s}} / \sigma$ & $\begin{array}{c}Y \\
(\%)\end{array}$ \\
\hline Dec $16 \ldots \ldots$ & $V$ & 0.0051 & 6.9 & Yes & 4.55 & 33.0 \\
& $R$ & 0.0027 & 6.9 & Yes & 8.01 & 25.6 \\
Dec $17 \ldots \ldots$ & $V$ & 0.0028 & 7.0 & Yes & 13.65 & 30.0 \\
& $R$ & 0.0030 & 7.0 & Yes & 12.35 & 28.5 \\
Dec $18 \ldots \ldots$ & $V$ & 0.0019 & 6.8 & Yes & 2.82 & 5.4 \\
& $R$ & 0.0025 & 6.8 & Yes & 1.71 & 3.7 \\
Dec $19 \ldots \ldots$ & $V$ & 0.0044 & 3.2 & Yes & 3.1 & 12.7 \\
& $R$ & 0.0066 & 3.2 & Yes & 3.0 & 17.3 \\
Dec $20 \ldots \ldots$ & $V$ & 0.0016 & 5.5 & No & $\ldots$ & $\ldots$ \\
& $R$ & 0.0028 & 5.5 & No & $\ldots$ & $\ldots$ \\
Dec $21 \ldots \ldots$ & $V$ & 0.0024 & 6.5 & Yes & 2.65 & 11.0 \\
& $R$ & 0.0028 & 6.5 & Yes & 2.65 & 11.5 \\
\hline
\end{tabular}

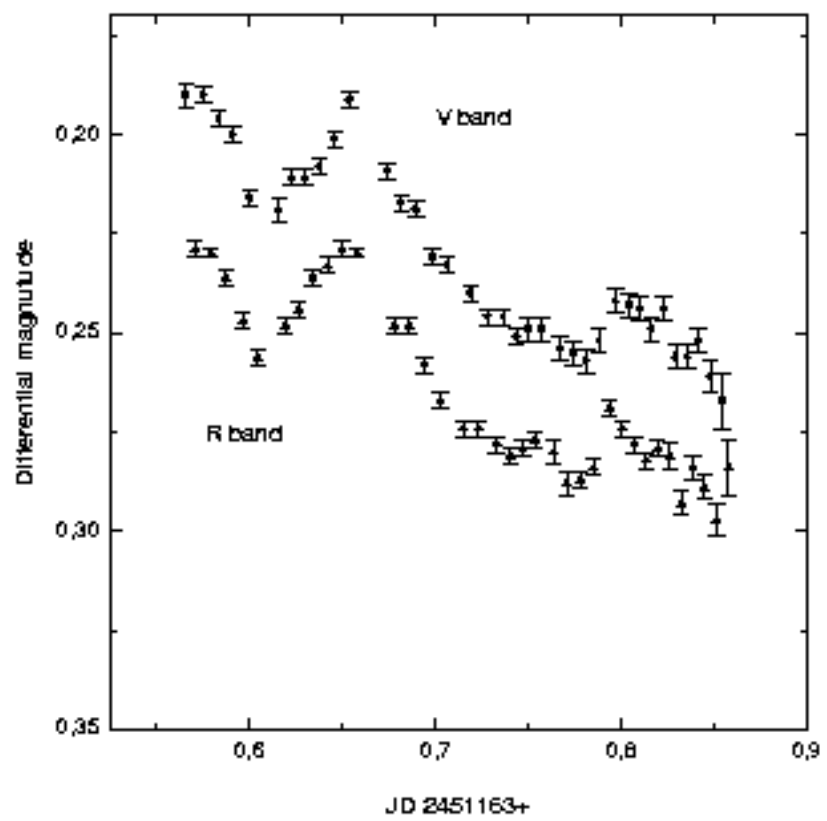

FIG. 3.-Differential light curves of PKS $0537-441$ for the first night of observation (1998 December 16).

\subsection{Spectral Behavior}

In Figure 6, we show the evolution of the spectral index $\alpha$ of PKS 0537-441 during the observations. Figure 7 presents a plot of the spectral index versus $V$ magnitude (corrected for reddening). The average value of the spectral index is $\langle\alpha\rangle=-1.6\left(F_{v} \propto v^{\alpha}\right)$. Fluctuations up to $|\Delta \alpha| \sim$ 0.44 are present, with a clear trend in the sense that the spectrum becomes steeper when the flux decreases. Pearson's correlation coefficient for the blazar (with the $R$ flux) is $r=0.67$, whereas the comparison star presents no

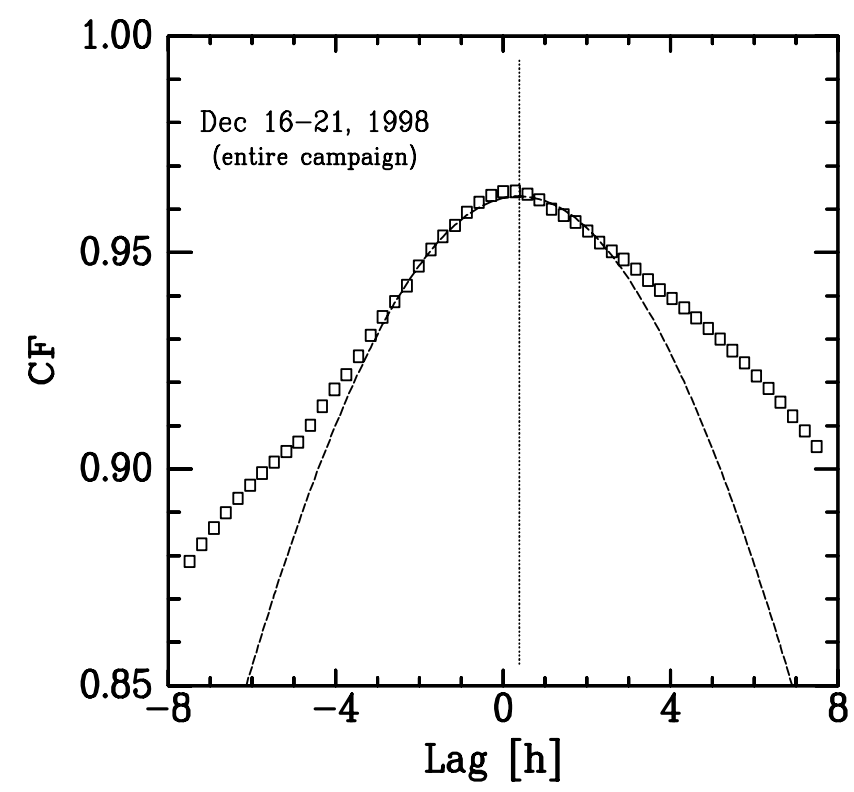

FIG. 4.- Interpolated correlation function for the entire campaign. The dashed line is the best Gaussian fit. The position of the maximum of the curve is indicated by a vertical line. 


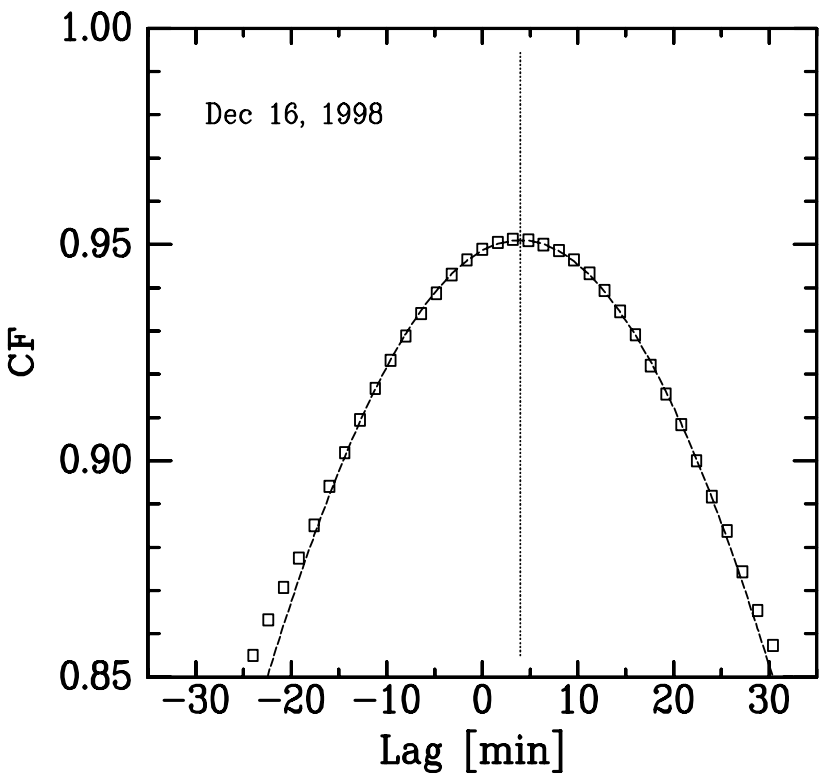

FIG. 5.-Same as Fig. 4, but for the first night of observation

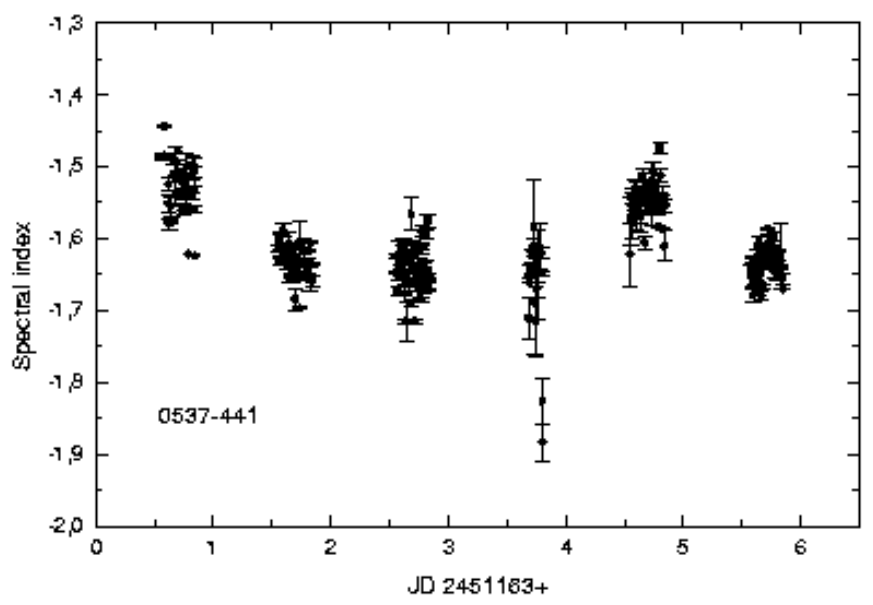

FIG. 6. - Spectral index behavior of PKS 0537-441 during the observations.

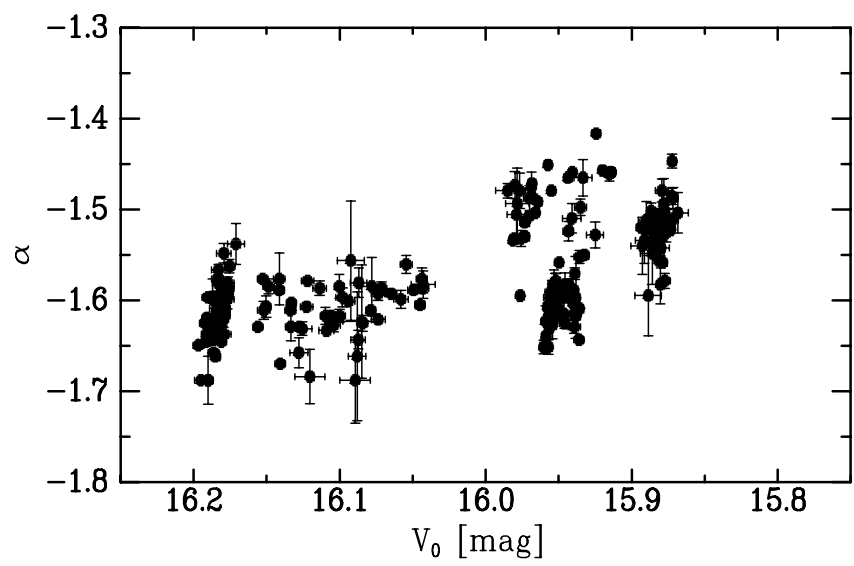

FIG. 7.- Spectral index vs. magnitude $V$ (corrected for Galactic reddening according to Schlegel, Finkbeiner, \& Davis 1998) for PKS 0537-441 during the entire campaign. correlation at all $(r=-0.02)$. Such behavior has been observed in other sources such as PKS 0735+178 (Fan, Lin, \& Xie 1997), PKS 2155 - 304 (Paltani et al. 1997), BL Lac (Fan et al. 1998), OJ 287 (Takalo \& Sillanpää 1989), and 3C 279 (Netzer et al. 1994; Bonnell, Vestrand, \& Stacy 1994), among others, but never before with such a short timescale as reported here. In the next section we shall discuss some implications of the observed behavior for the emission mechanisms in this source.

\section{DISCUSSION}

The observed variations in the spectral index of the optical emission of PKS 0537-441, with amplitudes of $\sim 27.5 \%$ in 2 days, seem to rule out gravitational microlensing (e.g., Romero et al. 1995) as a possible cause of the optical microvariability. Gravitational deflection of light is basically an achromatic phenomenon and should not affect the spectral index. In this sense, our observations support the arguments of Lewis \& Williams (1997) and the recent observations with the Hubble Space Telescope by Lewis \& Ibata (2000), which suggest that microlensing is not responsible for the large and fast outbursts observed in PKS 0537-441. Models in which the variability arises from purely geometric effects, such as shock propagation through swinging jets, where the Doppler factor changes with the variable line of sight (e.g., Gopal-Krishna \& Wiita 1992), should also be discarded by the same reason.

If the variability is produced in the accretion disk (e.g., by spiral shock fragmentation as suggested by Chakrabarti \& Wiita 1993 or by magnetic flares as proposed by Poutanen \& Fabian 1999), then beaming effects are not relevant and an upper limit to the size of the emitting region can be obtained through the variability timescale: $r \leq c t_{v} /(1+z)$. If this size is considered a bound on the possible gravitational radius of the central mass, we obtain from our observations that this mass should be $\leq 2.7 \times 10^{9} M_{\odot}$, assuming a Schwarzschild geometry $\left(r \sim 3 R_{q}=3 G M / c^{2}\right)$ and $t_{v}=$ $42.4 \mathrm{hr}$. This limit can be considerably lessened if the emission originates in a relativistic jet and beaming plays an important role. This latter possibility is more appealing for large flux variations than for accretion disk models, because Doppler boosting significantly reduces the energy requirements in the source.

Rapid optical variability can be produced when a relativistic shock wave propagates down a jet whose plasma is hydromagnetically turbulent (see, e.g., Marscher 1990; Marscher, Gear, \& Travis 1992). As the perturbation propagates along the jet, the optical synchrotron emission is enhanced at sites where the shock encounters particle or magnetic field overdensities. The amplitude and timescale of the resulting variability depend on the power spectrum of the turbulence and the shock thickness. The fact that very small and well-defined variations with timescales of $\sim 1 \mathrm{hr}$ are present in the light curve of PKS 0537-441 shows that, in this theoretical framework, the shock should be very thin and the emission should, consequently, be originating close to the central engine, before the shock has had time for significant broadening. This kind of shock-in-jet model quite naturally leads to the prediction that the spectrum steepens when the source becomes fainter, as observed in our case (e.g., Marscher 1998).

Estimates of the central black hole mass can be obtained using high-energy data. In particular, PKS 0537-441 has strong X-ray fields with a spectral index $\alpha_{\mathrm{X}}=-2.1 \pm 0.4$ 
(Treves et al. 1993). Gamma rays produced near the central black hole are absorbed in the radiation field by pair production processes, triggering a pair cascade that extends up to the place where the source becomes optically thin and the $\gamma$-emission can escape (see, e.g., Blandford \& Levinson 1995). For each photon energy there is a $\gamma$-sphere at which the radiation field becomes transparent to $\gamma$-ray propagation. If the Doppler factor is known, the observed $\gamma$-ray variability timescales can be used to set the size of the $\gamma$ spheres. Then the mass of the central black hole can be estimated if an emission model for the inner accretion disk region (which presumably generates the bulk of the X-ray emission) is adopted (e.g., Becker \& Kafatos 1995; Fan, Xie, \& Bacon 1999; Cheng, Fan, \& Zhang 1999).

In order to make some estimates in the case of PKS 0537-441, we shall follow the analytical treatment of Becker \& Kafatos (1995), who assumed that the inner disk emission can be represented by models in which the intensity has a dependence $I(E, R) \propto E^{\alpha \times} R^{-\xi}$, where $E$ is the energy and $R$ is the radial distance on the disk $\left(R_{\min } \leq R \leq\right.$ $R_{0}$ ). The parameter $\xi$ determines the kind of the disk emission structure: $\xi=3$ corresponds to two-temperature disks (e.g., Shapiro, Lightman, \& Eardley 1976), whereas $\xi=0$ corresponds to models with a single-temperature hot corona that cools by interactions with soft photons from an underlying cool disk (e.g., Liang 1979). Both kinds of model can reproduce the observed X-ray spectrum.

We have estimated the optical depth to pair creation for a $\gamma$-ray of energy $E=1 \mathrm{GeV}$ that propagates outward along the disk rotation axis for different models that are specified in Table 3. These models correspond to different central massive objects (Schwarzschild or Kerr black holes), different disk emission structures, and different outer radii for the $\mathrm{X}$-ray region. The size of the $\gamma$-spheres can be determined from the EGRET variability data (Hartman 1996) along with the Doppler factor $\delta \sim 3.8$ inferred by Fan et al. (1999). The observational high-energy parameters are listed in

TABLE 3

Central Black Hole Masses for Different Models of the Central Region in PKS 0537-441

\begin{tabular}{llcrc}
\hline \hline Model & \multicolumn{1}{c}{ BH Type } & $\begin{array}{c}\text { Disk } \\
\text { Type }\end{array}$ & $\begin{array}{c}R_{0} \\
\left(R_{g}\right)\end{array}$ & $\begin{array}{c}M \\
\left(10^{7} M_{\odot}\right)\end{array}$ \\
\hline A $\ldots \ldots$. & Schwarzschild & $\xi=3$ & 100 & 2.37 \\
B $\ldots \ldots$. & Schwarzschild & $\xi=0$ & 100 & 1.46 \\
C $\ldots \ldots$. & Schwarzschild & $\xi=3$ & 30 & 6.33 \\
D ..... & Schwarzschild & $\xi=0$ & 30 & 4.84 \\
E $\ldots \ldots$. & Kerr & $\xi=3$ & 100 & 3.09 \\
F $\ldots \ldots$. & Kerr & $\xi=0$ & 100 & 1.46 \\
G..... & Kerr & $\xi=3$ & 30 & 8.45 \\
H..... & Kerr & $\xi=0$ & 30 & 4.87 \\
\hline
\end{tabular}

Table 4. Then, by imposing that the optical depth must be equal to 1 since the emission is effectively observed, we obtain the masses of the central objects in the different models. These estimates are given in the last column of Table 3. In the case of a Kerr black hole with a twotemperature disk whose X-ray-emitting region extends up to $30 R_{g}$ (model G), a mass of $\sim 8.5 \times 10^{7} M_{\odot}$ is expected. The masses for Schwarzschild holes are somewhat lower but always above $10^{7} M_{\odot}$. Our estimates agree within an order of magnitude with those presented by Fan et al. (1999) and Cheng et al. (1999). It seems a common feature among rapidly variable $\gamma$-ray blazars to have relatively small central masses (see Fan et al. 1999). It is also worth mentioning that in the case of PKS 0537-441 the timescale of the large optical and $\gamma$-ray flux fluctuations is very similar ( $\sim 2$ days), highlighting the possibility that the emission at both energy ranges could be produced by the same particle population (Blandford \& Levinson 1995).

There remains the important point of whether there is a relation between the extremely rapid radio variability and the optical variations. Until now, no simultaneous observations of PKS 0537-441 at both bands have succeeded in recording a correlated activity. If such a correlation exists, the conclusion that coherent processes are responsible for at least a significant part of the emission could be unavoidable (Krishan \& Wiita 1990; Benford 1992; Romero et al. 1994; Wiita 1996; Benford \& Lesch 1998). If, instead, the evidence remains elusive, the most plausible picture would be that of a very compact source in which the optical variability is the result of synchrotron radiation in a relativistic shocked jet pointing nearly at the observer, and which exceptionally undergoes, as a result of its very small angular size, extreme scattering events produced by compact interstellar clouds, leading to isolated outbursts in the radio band at centimeter wavelengths. In any case, simultaneous multifrequency observations of this object will be a valuable tool to clarify the origin of the extreme forms of variability presented by some blazars.

This research has been supported by the Argentine agencies CONICET (under grants PIP No. 0430/98 and PEI No. 0151/98) and ANPCT (PICT 03-04881), as well as by Fundación Antorchas (funds granted to G. E. R. and J. A. C.). The authors acknowledge the use of the CCD and data acquisition system supported under US National Science Foundation grant AST 90-15827 to R. M. Rich. We are very grateful to the CASLEO staff for their kind assistance, to L. Mendoza for his help during the observations, and to L. Chajet for checking some numerical calculations. We also thank Professor Paul J. Wiita for useful discussions, and the referee, Professor Junhui Fan, for his valuable comments and suggestions on this work.

TABLE 4

Observational High-ENERgy Parameters for PKS 0537-441

\begin{tabular}{clll}
\hline \hline \multicolumn{1}{c}{ Parameter } & \multicolumn{1}{c}{ Symbol } & \multicolumn{1}{c}{ Value } & \multicolumn{1}{c}{ Reference } \\
\hline EGRET $\gamma$-ray flux ............ & $F_{\gamma}$ & $\sim 2 \times 10^{-6} \mathrm{~cm}^{-2} \mathrm{~s}^{-1}$ & Hartman 1996 \\
$\gamma$-ray variability timescale...... & $t_{v, \gamma}$ & $\sim 5.8 \times 10^{4} \mathrm{~s}$ & Fan et al. 1999 \\
ROSAT X-ray flux ............. & $F_{1 \mathrm{kev}}$ & $0.79 \pm 0.05 \mu \mathrm{Jy}$ & Treves et al. 1993 \\
X-ray spectral index ........... & $\alpha_{\mathrm{X}}$ & $-2.1 \pm 0.4$ & Treves et al. 1993 \\
Inferred Doppler factor ......... & $\delta$ & $\sim 3.8$ & Fan et al. 1999 \\
\hline
\end{tabular}


Becker, P. A., \& Kafatos, M. 1995, ApJ, 453, 83

Benford, G. 1992, ApJ, 391, L59

Benford, G., \& Lesch, H. 1998, MNRAS, 301, 414

Bessell, M. S. 1979, PASP, 91, 589

Blandford, R. D., \& Levinson, A. 1995, ApJ, 441, 79

Bonnell, J. T., Vestrand, W. T., \& Stacy, J. G. 1994, ApJ, 420, 545

Cellone, S. A., Romero, G. E., \& Combi, J. A. 2000, AJ, 119, 1534

Chakrabarti, S. K., \& Wiita, P. J. 1993, ApJ, 411, 602

Cheng, K. S., Fan, J. H., \& Zhang, L. 1999, A\&A, 352, 32

Eggen, O. J. 1973, ApJ, 186, L1

Fan, J. H., \& Lin, R. G. 2000, ApJ, 537, 101

Fan, J. H., Lin, R. G., \& Xie, G. Z. 1997, Ap\&SS, 249, 269

Fan, J. H., Xie, G. Z., \& Bacon, R. 1999, A\&AS, 136, 13

Fan, J. H., Xie, G. Z., Pecontal, E., Pecontal, A., \& Copin, Y. 1998, ApJ, 507,173

Gaskell, C. M., \& Peterson, B. M. 1987, ApJS, 65, 1

Gopal-Krishna \& Wiita, P. J. 1992, A\&A, 259, 109

Hartman, R. C. 1996, in ASP Conf. Ser. 110, Blazar Continuum Variabil-

ity, ed. H. R. Miller, J. R. Webb, \& J. C. Noble (San Francisco: ASP), 333

Heidt, J., \& Wagner, S. J. 1996, A\&A, 305, 42

Jang, M., \& Miller, H. R. 1995, ApJ, 452, 582 1997, AJ, 114, 565

Krishan, V., \& Wiita, P. J. 1990, MNRAS, 246, 597

Landolt, A. U. 1992, AJ, 104, 340

Lewis, G. F., \& Ibata, R. A. 2000, ApJ, 528, 650

Lewis, G. F., \& Williams, L. L. R. 1997, MNRAS, 287, 155

Liang, E. P. T. 1979, ApJ, 231, L111

Liller, W. 1974, ApJ, 189, L101
REFERENCES

Marscher, A. P. 1990, in Parsec-Scale Radio Jets, ed. J. A. Zensus \& T. J. Pearson (Cambridge: Cambridge Univ. Press), 236

. 1998, in Proc. OJ-94 Annu. Meeting 1997, Multifrequency Monitoring of Blazars, ed. G. Tosti \& L. O. Takalo (Publ. Oss. Astron. Univ Perugia, 3) (Perugia: Oss. Astron. Univ. Perugia), 81

Marscher, A. P., Gear, W. K., \& Travis, J. P. 1992, in Variability in Blazars, ed. E. Valtaoja \& M. Valtonen (Cambridge: Cambridge Univ. Press), 85 Netzer, H., et al. 1994, ApJ, 430, 191

Paltani, S., Courvoisier, T. J.-L., Blecha, A., \& Bratschi, P. 1997, A\&A, 327, 539

Peterson, B. A., Jauncey, D. L., Wright, A. E., \& Condon, J. J. 1976, ApJ, 207, L5

Poutanen, J., \& Fabian, A. C. 1999, MNRAS, 306, L31

Romero, G. E., Cellone, S. A., \& Combi, J. A. 1999, A\&AS, 135, 477

Romero, G. E., Combi, J. A., \& Colomb, F. R. 1994, A\&A, 288, 731

Romero, G. E., Surpi, G., \& Vucetich, H. 1995, A\&A, 301, 641

Schlegel, D. J., Finkbeiner, D. P., \& Davis, M. 1998, ApJ, 500, 525

Shapiro, S. L., Lightman, A. P., \& Eardley, D. M. 1976, ApJ, 204, 187

Surpi, G. C., Romero, G. E., \& Vucetich, H. 1996, Rev. Mexicana Astron. Astrofis., 32, 153

Takalo, L. O., \& Sillanpää, A. 1989, A\&A, 218, 45

Tanzi, E. G., et al. 1986, ApJ, 311, L13

Thompson, D. J., et al. 1993, ApJ, 410, 87

Tingay, S. J., et al. 1996, ApJ, 464, 170

Treves, A., Belloni, T., Falomo, R., Fink, H., Maraschi, L., Sambruna, R. M., Tagliaferri, G., \& Zimmermann, H. U. 1993, ApJ, 406, 447

Wiita, P. J. 1996, in ASP Conf. Ser. 110, Blazar Continuum Variability, ed. H. R. Miller, J. R. Webb, \& J. C. Noble (San Francisco: ASP), 42 